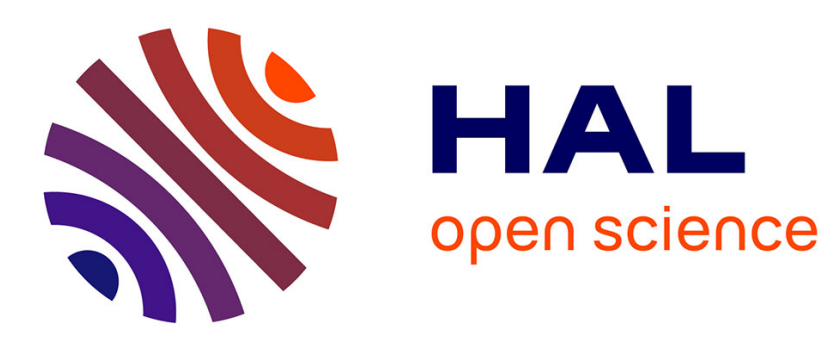

\title{
Internal Structure of Inertial Granular Flows
}

Emilien Azéma, Farhang Radjai

\section{To cite this version:}

Emilien Azéma, Farhang Radjai. Internal Structure of Inertial Granular Flows. Physical Review Letters, 2014, 12, pp.078001. 10.1103/PhysRevLett.112.078001 . hal-00957291

\section{HAL Id: hal-00957291 \\ https://hal.science/hal-00957291}

Submitted on 10 Mar 2014

HAL is a multi-disciplinary open access archive for the deposit and dissemination of scientific research documents, whether they are published or not. The documents may come from teaching and research institutions in France or abroad, or from public or private research centers.
L'archive ouverte pluridisciplinaire HAL, est destinée au dépôt et à la diffusion de documents scientifiques de niveau recherche, publiés ou non, émanant des établissements d'enseignement et de recherche français ou étrangers, des laboratoires publics ou privés. 


\title{
Internal structure of inertial granular flows
}

\author{
Emilien Azéma and Farhang Radjaï \\ Université Montpellier 2, CNRS, LMGC, Cc 048, \\ Place Eugène Bataillon, F-34095 Montpellier cedex 05, France*
}

(Dated: December 18, 2013)

\begin{abstract}
We analyze inertial granular flows and show that, for all values of the inertial number $I$, the effective friction coefficient $\mu$ arises from three different parameters pertaining to the contact network and force transmission: 1) contact anisotropy, 2) force chain anisotropy and 3) friction mobilization. Our extensive 3D numerical simulations reveal that $\mu$ increases with $I$ mainly due to an increasing contact anisotropy and partially by friction mobilization whereas the anisotropy of force chains declines as a result of the destabilizing effect of particle inertia. The contact network undergoes topological transitions, and beyond $I \simeq 0.1$ the force chains break into clusters immersed in a background "soup" of floating particles. We show that this transition coincides with the divergence of the size of fluidized zones characterized from the local environments of floating particles and a slower increase of $\mu$ with $I$.
\end{abstract}

PACS numbers: 45.70.-n,83.80.Fg, 45.70.Mg, 81.05.Rm

From large-scale geological events to a variety of industrial processes involving bulk materials and powders, the flow behavior of granular materials has been a subject of intensive research since many years [1-6]. The diversity of boundary conditions and confining geometries made it difficult for a long time to extract the intrinsic rheology of dense inertial flows until a unification was achieved by analyzing several experimental and numerical data in terms of a single dimensionless inertial number $I$, defined as the ratio of the particle relaxation time $(m / p d)^{1 / 2}$, under an average or confining stress $p$ and for a particle of mass $m$ and diameter $d$, to shear time $\dot{\gamma}^{-1}$ imposed by the flow rate $\dot{\gamma}[7,8]$. The model arising from this seminal work is based on a generic dependence of the effective friction coefficient $\mu$ and packing fraction $\nu$ on $I$.

This empirical model of steady granular flows, in combination with continuum conservation equations, correctly predicts the velocity and stress fields in various flow geometries [7, 9-11]. However, it still lacks a clear particle-scale foundation. The increase of $\mu$ with $I$ despite an increasingly lower packing fraction is a nontrivial property that reveals a genuine microstructure. A few studies reported on the microstructure of inertial flows $[8,12-15]$ show that, as $I$ increases, the force chains become more sparse, the correlation length of connected particles decrease $[5,16,17]$, the contact lifetimes decline, and an increasing number of impulsive forces $[18,19]$ and frictionally mobilized contacts $[4,8]$ come into play. But a challenging issue is how to connect such particle-scale observations with the rheology.

In this Letter, we analyze inertial granular flows by means of a stress partition that readily links the $\mu(I)$ rheology to three different particle-scale mechanisms: 1) contact anisotropy, 2) force chain anisotropy and 3) fric-

\footnotetext{
*Electronic address: emilien.azema@univ-montp2.fr;franck. radjai@univ-montp2.fr
}

tion mobilization. The data are obtained from extensive contact dynamics simulations of homogeneous shear flow with spherical particles for a broad range of $I$ varied from low to very high values. Stress partition has been successfully applied to quasi-static deformations [20-24] but never to inertial flows. As we shall see below, the relative importance of local mechanisms evolves with $I$ and therefore the flow structure undergoes qualitative transitions that underlie the evolution of the effective friction. Such transitions are consistent with the correlative evolution of the statistics of fluidized zones that we analyze by considering the clustering of floating particles.

Contact dynamics simulations were carried out with $N_{p}=24000$ spheres. The packing is sheared between two parallel rough walls by imposing a constant horizontal velocity $V_{y}$ on the top wall and periodic boundary conditions along the flow in the $y$ direction and along the transversal $x$ direction. The packing is confined by a constant compressive stress $\sigma_{z z}$ applied on the top wall along the $z$-direction; see Fig.5(a). A small polydispersity $(1 \pm 0.1) d$ in particle diameters is introduced to prevent crystallization at the walls. The gravity is set to zero in order to avoid strain localization at the boundaries and to ensure uniform stress field in the bulk. In the contact dynamics method, the particles are treated as perfectly rigid so that $I$ is the only relevant dimensionless parameter of flow [24-26]. Contact dissipation is modeled in terms of normal and tangential restitution coefficients $e_{n}$ and $e_{t}$ as well as a friction coefficient $\mu_{s}$ between particles. We set $\mu_{s}=0.4$ and $e_{n}=e_{t}=0$. This choice corresponds to a highly dissipative packing. We note that the values of restitution coefficients have nearly no influence on dense granular flows as inelastic collisions occur at high frequency and dissipate the kinetic energy at time scales far shorter than those of shear and particle relaxation $[7,8]$.

Under the action of the applied shear strain, all samples dilate from their initial high density and tend to a steady flow characterized by a linear velocity profile. We 
performed 17 simulations for a broad range of $I$ varied from $6 \times 10^{-4}$ to 0.72 by keeping the same shear rate and changing the confining pressure [36]. The data presented in this Letter, are average values over the steady state with standard deviations used as error bars. The values of $I$ are obtained from the average stress $p$, which fluctuates in the steady state around the average stress. The error bars on the values of $I$ represent these fluctuations. Obviously, the fluctuations increase with $I$, and thus the data are to be sampled more frequently in the steady state in order to reach meaningful statistics.

The stress state being invariant along the $x$ direction, we consider here only the restriction $\boldsymbol{\sigma}$ of the stress to the shear plane $y z$. It may be expressed as [27]

$$
\sigma_{\alpha \beta}=n_{c}\left\langle f_{\alpha}^{c} \ell_{\beta}^{c}\right\rangle
$$

where $n_{c}$ is the number density of the contacts and the average is taken over the contacts $c$ with contact force component $f_{\alpha}^{c}$ and branch vector $\ell_{\beta}^{c}$ joining the centers of contacting particles. Note that, the contribution of particle velocity fluctuations $\left(\left\langle m v_{\alpha} v_{\beta}\right\rangle / V\right)$ remains very small compared to that of contact forces in all the simulations considered here. According to the Mohr-Coulomb model, the effective friction coefficient during shear is given by $\mu \simeq q / p$, where $p=\left(\sigma_{1}+\sigma_{2}\right) / 2$ is the mean stress and $q=\left(\sigma_{1}-\sigma_{2}\right) / 2$ is the stress deviator, and $\sigma_{1}$ and $\sigma_{2}$ are the principal values on the shear plane [28].

Fig. 1 displays $\mu$ and $\nu$ obtained from our simulations and a compilation of available published numerical and experimental data from several authors for different boundary conditions as a function of $I[1-5,29]$ [37]. We see that our numerical data collapse well with all other data. The effective friction coefficient increases and tends to saturate with increasing $I$ whereas the packing fraction declines from 0.59 in the quasi-static state to 0.50 for our highest values of $I$. Note that 0.59 corresponds to the density of a packing of frictional spheres under continuous quasi-static shearing, as also evidenced by experiments [30].

Relying on our numerical data, we now focus on the stress partition in connection with the effective friction. Let us express the average in (1) as an integral:

$$
\sigma_{\alpha \beta}=n_{c} \iiint f_{\alpha} \ell_{\beta} P_{\ell f n} d \boldsymbol{f} d \boldsymbol{\ell} d \boldsymbol{n},
$$

where $P_{\ell f n}$ is the joint probability density of forces and branch vectors $\boldsymbol{\ell}=\boldsymbol{\ell} \boldsymbol{n}$ projected on the shear plane. At the lowest-order description of the microstructure, we neglect the force-fabric correlations and split $P$ as a product $P_{\ell f n}=P_{\ell}(\boldsymbol{\ell}) P_{f}(\boldsymbol{f}) P_{n}(\boldsymbol{n})$. Integration over $\boldsymbol{f}$ and $\boldsymbol{\ell}$ yields:

$$
\sigma_{\alpha \beta} \simeq n_{c} \ell_{0} \int_{\Omega}\left\langle f_{\alpha}\right\rangle(\boldsymbol{n}) n_{\beta} P_{n}(\boldsymbol{n}) d \boldsymbol{n},
$$

where $\Omega$ is the angular domain of integration and $\langle\boldsymbol{f}\rangle(\boldsymbol{n})$ is the average force as a function of $\boldsymbol{n}$ and $\langle\ell\rangle=\ell_{0}$.
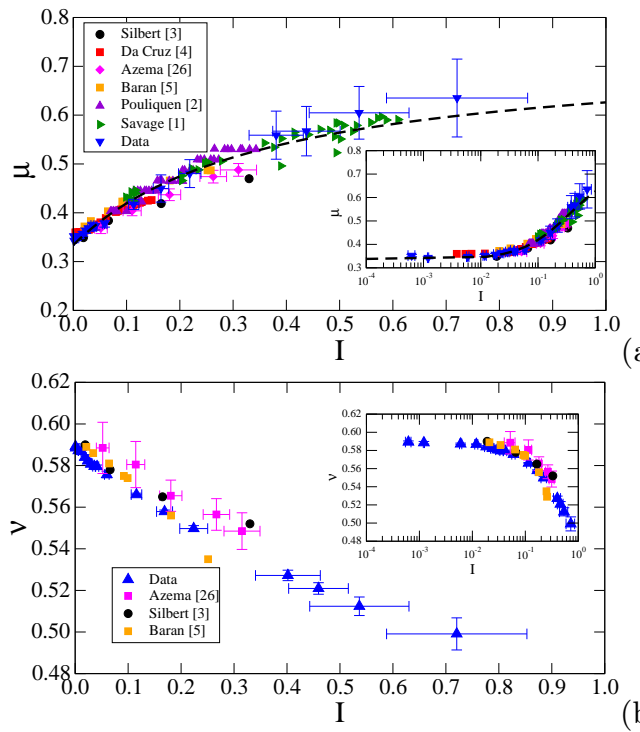

FIG. 1: (Color online) Effective friction coefficient (a) and packing fraction (b) as a function of $I$. The data analyzed in this paper are in blue triangles. The other data are extracted from $[1-5,29]$. The dashed line shows the fitting form $\mu=$ $\mu_{0}+\frac{\mu_{1}-\mu_{0}}{1+I_{0} / I}$ introduced in $[9,10]$.

The contact force on the shear plane can be decomposed into its normal and tangential components $\left\langle f_{n}\right\rangle(\boldsymbol{n})$ and $\left\langle f_{t}\right\rangle(\boldsymbol{n})$, and $\boldsymbol{n}$ is parametrized by its orientation $\theta$. The three functions $P(\theta),\left\langle f_{n}\right\rangle(\theta)$ and $\left\langle f_{t}\right\rangle(\theta)$ are $\pi$ periodic and, as shown in Fig.3, they can be well approximated by their lowest-order Fourier expansions [20$23,31]$ :

$$
\begin{aligned}
P(\theta) & \simeq 1 / \pi\left\{1+a_{c} \cos 2\left(\theta-\theta_{c}\right)\right\} \\
\left\langle f_{n}\right\rangle(\theta) & \simeq\left\langle f_{n}\right\rangle\left\{1+a_{n} \cos 2\left(\theta-\theta_{n}\right)\right\} \\
\left\langle f_{t}\right\rangle(\theta) & \simeq-\left\langle f_{n}\right\rangle a_{t} \sin \left(\theta-\theta_{t}\right)
\end{aligned}
$$

where $a_{c}, a_{n}$, and $a_{t}$ are anisotropy parameters, and $\theta_{c} \simeq \theta_{n} \simeq \theta_{t}$ are the corresponding privileged directions on the shear plane, and nearly coincide with the major principal stress direction in the steady state. Now, introducing Eqs. (4) in the integral (2), and neglecting the cross products of the anisotropy parameters, one gets the simple relation:

$$
\mu \simeq \frac{1}{2}\left(a_{c}+a_{n}+a_{t}\right)
$$

The predicted values of $\mu$ by this equation from the anisotropy parameters are shown in Fig. 2 together with those obtained from the stress tensor as a function of $I$. We see that Eq. (5) approximates excellently the effective friction for all values of $I$. This result indicates also that the expression (1) of the stress tensor holds correctly for high inertia where impulsive forces prevail.

The evolution of the three anisotropies with $I$ is plotted in Fig.3. Interestingly, the normal force anisotropy $a_{n}$ decreases and tends to a constant value whereas the friction force anisotropy slightly increases with $I$. At the 


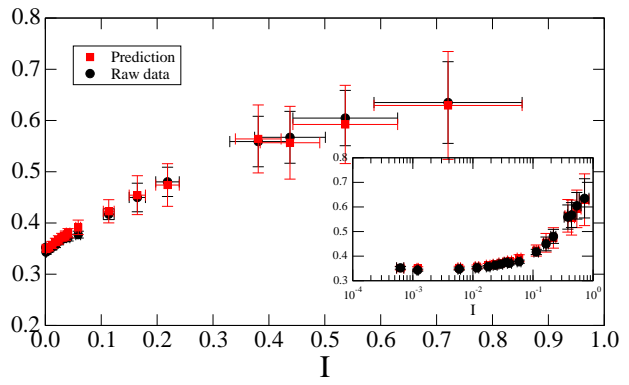

FIG. 2: (Color Online) Effective friction coefficient $\mu$ (black circles) together with its harmonic approximation (Eq.5) (red squares) as a function of inertial number $I$.

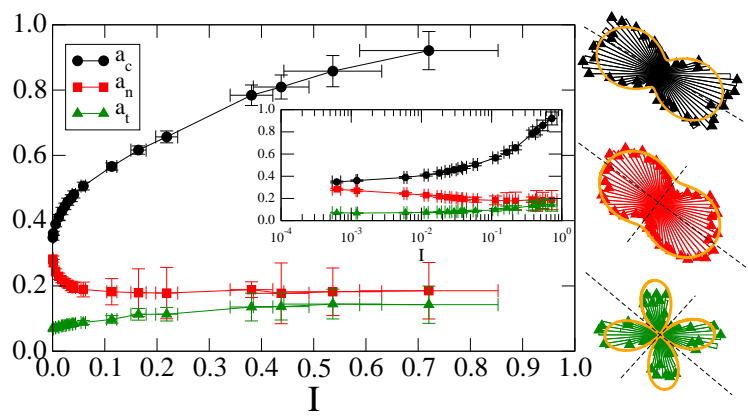

FIG. 3: (Color online) Evolution of anisotropy parameters as a function of $I$ in log-linear and linear representation. The polar diagrams of $P_{n}(\theta)$ (black circle), $\left\langle f_{n}\right\rangle(\theta)$ (red square) and $\left\langle f_{t}\right\rangle(\theta)$ (green triangle) are shown for $I \sim 0.1$ together with their fits (plain orange line) (Eq. 4).

same time, the contact anisotropy $a_{c}$ is a sharply increasing and nonlinear function of $I$. This means that, since by virtue of Eq. (5) the three anisotropies add up to build the effective friction, the contact anisotropy is the principal microstructural cause of the increase of effective friction as a function of the inertial number. In general, $a_{c}$ varies oppositely with the coordination number $z$, which declines in our simulations from 4.5 to 1.1 as $I$ varies from $10^{-4}$ to 0.7 . This is because the contact anisotropy is mainly a consequence of the loss of contacts in the extension direction [32]. The normal force anisotropy $a_{n}$ reflects the force chains, which are increasingly destabilized by particle inertia causing $a_{n}$ to decrease. Hence, the friction force anisotropy $a_{t}$, which reflects friction mobilization $\left(\left\langle\left|f_{t}\right|\right\rangle /\left\langle f_{n}\right\rangle \propto a_{t}\right)$, grows to re-stabilize the force chains and thus take more actively part in force transmission [23].

Since the contact anisotropy and coordination number seem to be sensibly important with respect to the effective friction, we now turn to the connectivity of the contact network in order to obtain morphological clues to the evolution of the force network. The connectivity can be described by the proportion $P_{k}$ of force-bearing particles having $k \geq 1$ contacts and the proportion $P_{0}$ of floating particles, i.e. not participating in the force

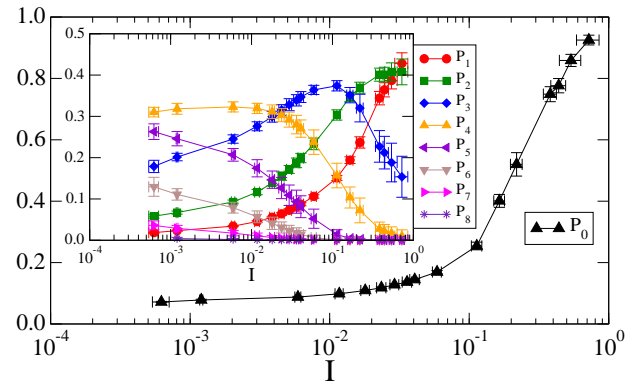

FIG. 4: (Color online) Proportion of floating particles $P_{0}$ and the connectivity $P_{k}$ of the particles (inset) as a function of $I$.

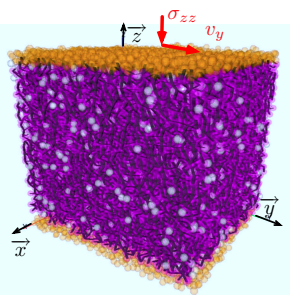

(a)

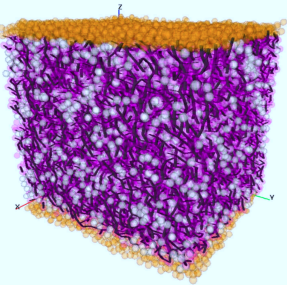

(b)
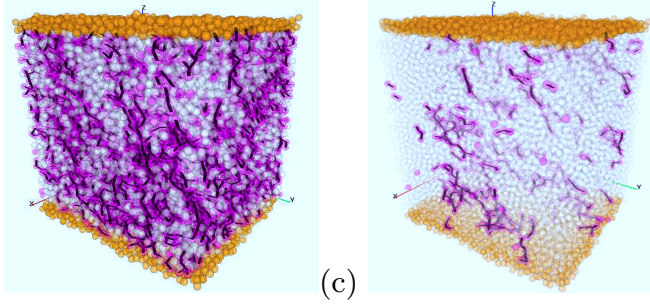

(d)

FIG. 5: (Color online) Floating particle are in grey, nonfloating in violet for $I \sim 10^{-3}(\mathrm{a}), I \sim 0.1(\mathrm{~b}), I \sim 0.21(\mathrm{c})$ and $I \sim 0.6(\mathrm{~d})$. Black lines represents the connection between particles (i.e. the length of force chains).

network. Note that $\sum_{k>1} P_{k}=1$ and $\sum_{k>1} k P_{k}=z$. Fig. 4 displays $P_{k}$ for $k=1$ to 8 and $P_{0}$ as a function of $I$. We observe several nontrivial topological transitions. For $I<0.01$, the effect of inertia leads to the reduction of highly-connected particles $(k>4)$ in favor of low-connected particles $(k<4)$ while $P_{4}$ remains nearly constant. In this range, $P_{0} \simeq 0.07$. At higher values of $I$, the force network is further destabilized and $P_{4}$ begins to decline whereas $P_{3}$ keeps increasing up to a peak value at $I \simeq 0.1$ beyond which $P_{3}$ also begins to decline whereas $P_{2}$ continues to increase. The loss of particles having 3 contacts is a dramatic change in the microstructure as multiple contacts may thereafter occur mainly in the form of linear force chains without branching, as observed in Fig. 6(d). $P_{0}$ increases slightly in this range from 0.07 to 0.2 but undergoes a sharp increase beyond $I \simeq 0.1$. For $I>0.3$, the flow is dominated by $P_{2}$ and $P_{1}$ (corresponding to the collisions of floating particles).

The sharpt transition observed at $I \simeq 0.1$ for $P_{0}$ coincides with the slowing down of $\mu(I)$; see Fig. 1 on linear scale. At the same time, the normal force anisotropy ceases to decline and takes a constant value $a_{n} \simeq 0.2$; see Fig. 3. The fact that beyond $I \simeq 0.1$ the force anisotropy 


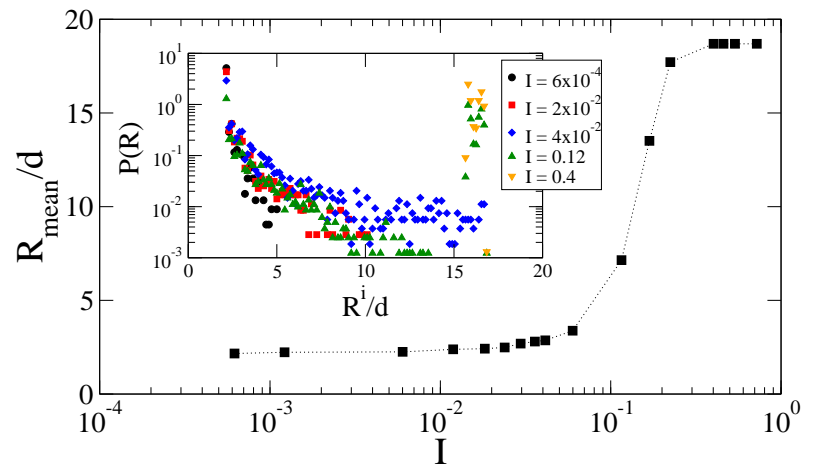

FIG. 6: Mean size of critical floating clusters as a function of $I$. The inset shows the statistical distributions of $R^{i}$ for several values of $I$.

does not follow the contact anisotropy indicates that the force chain formation during flow is hindered by collisions, and the contacts do not live long enough to sustain percolating force chains. Indeed, at $I \simeq 0.1$ a fraction $P_{0}^{*}=0.2$ of particles is floating and this fraction of particles disconnected from the force network is consistent with the site percolation threshold of a hexagonal compact packing [33]. This means that, beyond $I \simeq 0.1$, the effect of inertia may be analyzed more sensitively in terms of the evolution of floating particles rather than connected chains, whose correlations have already been a subject of several studies to characterize the transition from inertial regime to quasi-static regime $[13,16]$.

The floating particles actually provide a complementary picture in terms of fluidized zones defined from the neighborhoods of floating particles. For each floating particle $i$, let $P_{0}^{i}(I, R)$ be the proportion of floating particles for a spherical volume of radius $R$ centered on $i$. We determine the size $R^{i}$ of the fluidized zone by requiring $P_{0}^{i}\left(I, R^{i}\right)=P_{0}^{*}$. In words, this is the size of the spherical volume in which the density of floating particles is equal to the percolation threshold $P_{0}^{*}$. The distribution $P(R)$ of the sizes of fluidized zones for given value of $I$ can be evaluated from the set $\left\{R^{i}\right\}$ and, their mean size $R_{\text {mean }}$ is determined by double averaging $R^{i}$ over all floating particles and during shear. This construction is nearly dual to the 2-point cluster function introduced by Torquato et al. $[34,35]$.

Figure 5 shows four snapshots of floating particles for different values of $I$. At low $I$ the floating particles are mostly isolated, but they tend to cluster into fluidized zones as $I$ increases. Fig. 6 displays the clustering length $R_{\text {mean }}$ as a function of $I$. The inset shows the statistical distributions of $R^{i}$ for several values of $I$. We see that $R_{\text {mean }}$ is nearly constant and equal to $2 d$ for $I<0.1$, as expected for isolated floating particles. Thereafter, $R_{\text {mean }}$ grows rapidly with $I$ and reaches the system size for $I \simeq 0.3$. This evolution reflects the coalescence process of fluidized zones and a transition to the collisional regime at $I \simeq 0.3$ where $P_{0}>0.6$. This process is also clearly evidenced by the evolution of the size distributions of fluidized zones, which broaden as $I$ increases up to $I \simeq 0.1$. For $I>0.1$, the distribution develops a second peak for $R$ equal to the system size whereas the mean size of the fluidized zones continues to increase.

To summarize, in the range $I<0.1$ the force anisotropy reflects percolating force chains, which are progressively destabilized by inertial effetcs with increasing $I$. As a result, the force anisotropy declines towards a residual constant value $a_{n} \simeq 0.2$ for $I>0.1$. This residual force anisotropy is essentially induced by collisions due to shearing, which begin to affect the microstructure in the form of fluidized zones of increasing size that coalesce at $I=0.3$. Hence, in the whole range $I>0.1$, the microstructure may be described as composed of shortlived and impulsive force chains embedded in a "soup" of floating particles. The contact anisotropy grows due to enduring force chains and by loss of contacts for $I<0.1$ and due to both impulsive force chains and shear-induced collisions of floating particles beyond $I=0.1$. This geometrical anisotropy provides the main additive contribution to the effective shear friction of the flow according to Eq. (5). The transition to a fully collisional regime occurs at $I \simeq 0.3$ where the whole system is in a fluidized state. Let us also note that the value $I=0.3$ may be identified with the reference value $I_{0}$ in the fitting form introduced in $[9,10]$ and shown in Fig. 1.

The above picture reveals the highly nonlinear evolution of the microstructure with increasing inertial number that we analyzed in terms of three anisotropy parameters, which underly additively the effective friction. The dynamics of local structures may be further investigated by considering in detail the contact lifetimes, the process of contact gain and loss and the role of impulsive forces, as discussed in $[18,19]$ regarding the jamming transition in a suspension.
[1] S. Savage, Adv. Appl. Mech. 24, 289 (1984).

[2] O. Pouliquen, Phys. Fluids 11, 542 (1999).

[3] L. Silbert, D. Ertas, G. Grest, T. C. Halsey, D. Levine, and S. J. Plimpton, Phys Rev E 64, 051302 (2001).

[4] F. da Cruz, Ph.D. thesis, Ecole Nationale des Ponts et Chaussées (2004), http://pastel.paristech.org/946.

[5] O. Baran, D. Ertas, T. Halsey, G. Grest, and J. Lechman, Phys Rev E 74, 05130200 (2006).
[6] T. Borzsonyi and R. Ecke, Phys Rev E 76, 031301 (2007).

[7] GDR-MiDi, Eur. Phys. J. E 14, 341 (2004).

[8] F. da Cruz, S. Emam, M. Prochnow, J.-N. Roux, and F. Chevoir, Phys. Rev. E 72, 021309 (2005).

[9] P. Jop, Y.Forterre, and O.Pouliquen, Nature 441, 727 (2006).

[10] Y. Forterre and O. Pouliquen, Annu. Rev. Fluid Mech. 40, 1 (2008). 
[11] K. Kamrin and G. Koval, Phys Rev Letter 108, 178301 (2012).

[12] L. E. Silbert, G. Grest, R. Brewster, and A. Levine, Phys Rev. Letter 99, 068002 (2007).

[13] P. Mills, P. G. Rognon, and F. Chevoir, Eur. Phys. Letter 81, 64005 (2008).

[14] P.-E. Peyneau and J.-N. Roux, Phys Rev E 78, 041307 (2008).

[15] X. Wang, H. P. Zhu, S. Luding, and A. B. Yu, Phys Rev E 88, 032203 (2013).

[16] D. Bonamy, F. Daviaud, L. Laurent, M. Bonetti, and J. P. Bouchaud, Physical Review Letters 89 (2002).

[17] O. Pouliquen, Physical Review Letters 93, 248001 (2004).

[18] G. Lois, A.Lemaitre, and J. M. Carlson, Phys Rev E 76, 021302 (2007).

[19] G. Lois, A.Lemaitre, and J. M. Carlson, Phys Rev E 76, 021303 (2007).

[20] L. Rothenburg and R. J. Bathurst, Geotechnique 39, 601 (1989).

[21] H. Ouadfel and L. Rothenburg, Mechanics of Materials 33, 201 (2001).

[22] E. Azéma, F. Radjai, and G. Saussine, Mechanics of Materials 41, 721 (2009).

[23] E. Azéma, F. Radjai, and F. Dubois, Phys Rev E 87, 062203 (2013).

[24] E. Azéma, N. Estrada, and F. Radjai, Phys Rev E 86, 041301 (2012).

[25] J. Moreau, Eur. J. Mech. A Solids 13, 93 (1994).

26] F. Radjaï and V. Richefeu, Mechanics of Materials 41, 715 (2009).

[27] J. J. Moreau, in Friction, Arching, Contact Dynamics, edited by D. E. Wolf and P. Grassberger (World Scientific, Singapore, 1997), pp. 233-247.

[28] B. Andreotti, Y. Forterre, and O. Pouliquen, Granular Media: Between Fluid and Solid (Cambridge University Press, 2013).

[29] E. Azéma, Y. Descantes, N. Roquet, J.-N. Roux, and F. Chevoir, Phys Rev E 86, 031303 (2012).

[30] M. Pailha, M. Nicolas, and O. Pouliquen, PHYSICS OF FLUIDS 20, 111701 (2008).

[31] C. Voivret, F. Radjai, J.-Y. Delenne, and M. S. E. Youssoufi, Phys. Rev. Lett. 102, 178001 (2009).

[32] F. Radjai, J.-Y. Delenne, E. Azéma, and S. Roux, Granular Matter Doi 10.1007/s10035-012-0321-8 (2012).

[33] C. D. Lorenz, R. May, and R. Ziff, Journal of Statistical Physics 98, Issue 3-4, 961 (2000).

[34] S. Torquato, J. Beasley, and Y. Chiew., J . Chern. Phys 88, 6540 (1988).

[35] S. Torquato, Random heterogeneous materials, vol. 16 of Interdisciplinary Applied Mathematics (S.S. Antman and J.E. Marsden and L. Sirovich and S. Wiggins, 2002), springer ed.

[36] Video samples of the simulations analyzed in this Letter can be found following this link: www.cgpgateway.org/ref025

[37] The compiled data $[1,2,5]$ are extracted from [10] 